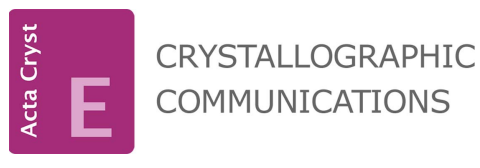

ISSN 2056-9890

Received 28 May 2015

Accepted 23 June 2015

Edited by A. J. Lough, University of Toronto, Canada

Keywords: crystal structure; terpyridine; nitroxide; nitroxyl; $\mathrm{C}-\mathrm{H} \cdots \pi$ interactions; $\pi-\pi$ interactions; $\mathrm{C}-\mathrm{H}$... O hydrogen bonding

CCDC reference: 1408457

Supporting information: this article has supporting information at journals.iucr.org/e

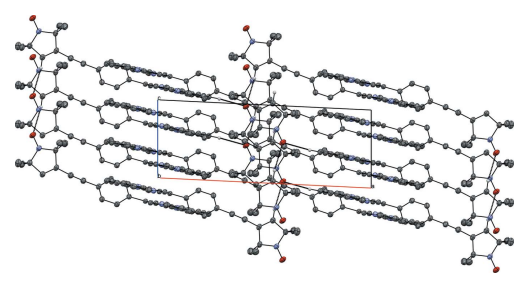

OPEN $\odot$ ACCESS

\section{The crystal structure of $4^{\prime}-\{4-[(2,2,5,5$-tetramethyl- $\mathrm{N}$-oxyl-3-pyrrolin-3-yl)ethynyl]phenyl\}-2,2':6', $2^{\prime \prime}-$ terpyridine}

\author{
Andreas Meyer, ${ }^{a}$ Jennifer Wiecek, ${ }^{a}$ Gregor Schnakenburg ${ }^{\mathrm{b}}$ and Olav Schiemann ${ }^{\mathrm{a} *}$ \\ a University of Bonn, Institute of Physical and Theoretical Chemistry, Wegelerstrasse 12, 53115 Bonn, Germany, and \\ ${ }^{b}$ University of Bonn, Institute of Inorganic Chemistry, Gerhard-Domagk-Strasse 1, 53121 Bonn, Germany. \\ *Correspondence e-mail: schiemann@pc.uni-bonn.de
}

The terpyridine group of the title compound, $\mathrm{C}_{31} \mathrm{H}_{27} \mathrm{~N}_{4} \mathrm{O}$, assumes an alltransoid conformation and is essentially planar with the dihedral angles between the mean planes of the central pyridine and the two outer rings amounting to $3.87(5)$ and $1.98(5)^{\circ}$. The pyrroline- $N$-oxyl group commonly seen in such nitroxyls is found in the title structure and the mean plane of the pyrroline ring subtends a dihedral angle of $88.44(7)^{\circ}$ to the mean plane of the central pyridine ring. The intramolecular separation between the nitrogen atom of the central pyridine unit of the terpyridine group and the nitroxyl group is 14.120 (2) $\AA$. In the crystal, the molecules are arranged in layers stacked along [001]. Slipped face-to-face $\pi-\pi$ interactions between the pyridine rings are observed along this direction with the shortest centroid-centroid distances amounting to 3.700 (1) and 3.781 (1) A. Furthermore, edge-on $\mathrm{C}-\mathrm{H} \cdots \pi$ interactions between the phenylene rings of neighbouring molecules are observed along this direction. A two-dimensional $\mathrm{C}-\mathrm{H} \cdots \mathrm{O}$ hydrogen-bonded network is formed within the (010) plane. The shortest $\mathrm{O} \ldots \mathrm{O}$ separation between neighbouring molecules is $5.412(3) \AA$.

\section{Chemical context}

The title compound, (1), was synthesized as a ligand for $3 d$ metal ions as part of a pulsed EPR study on metal-nitroxyl model systems. The molecule contains a paramagnetic nitroxyl group and a terpyridine group. Nitroxyls have been the subject of magnetic studies in which exchange interactions have been detected (see, for example, Rajca et al., 2006; Fritscher et al., 2002). Furthermore, nitroxyls are used as spin labels for structural investigations of biological macromolecules (Reginsson \& Schiemann, 2011). The structures of terpyridines have been investigated by Fallahpour et al. (1999), Eryazici et al. (2006), Bessel et al. (1992) and Grave et al. (2003) to name a few examples. The terpyridine moiety is known to form complexes with various metals. Numerous studies on metal complexes of terpyridine have been conducted, examples include those by Hogg \& Wilkins (1962), Constable et al. (1999), Narr et al. (2002) and Folgado et al. (1990).

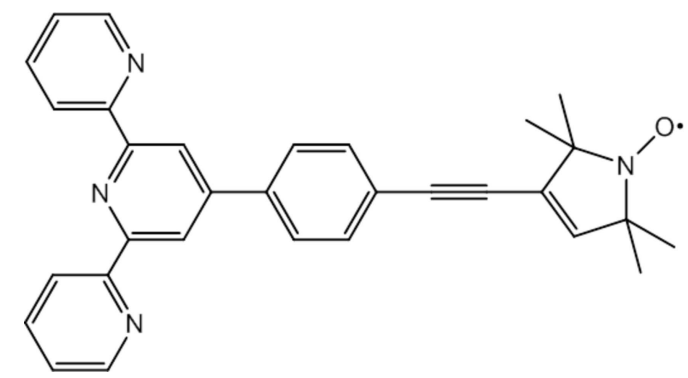




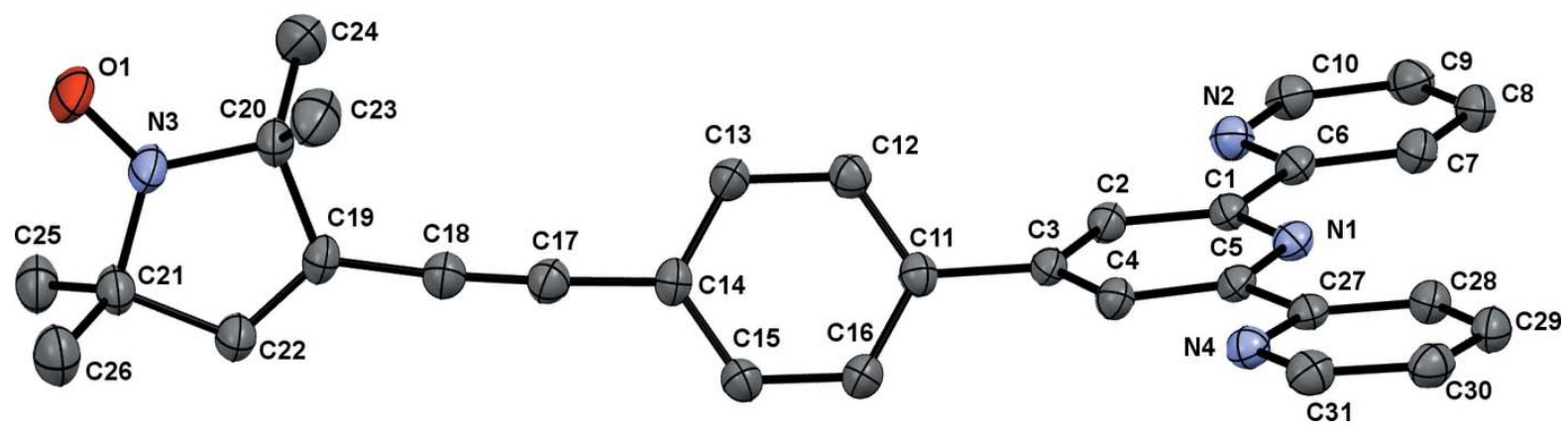

Figure 1

The molecular structure of the title compound with displacement ellipsoids drawn at the $50 \%$ probability level. $\mathrm{H}$ atoms have been omitted for clarity.

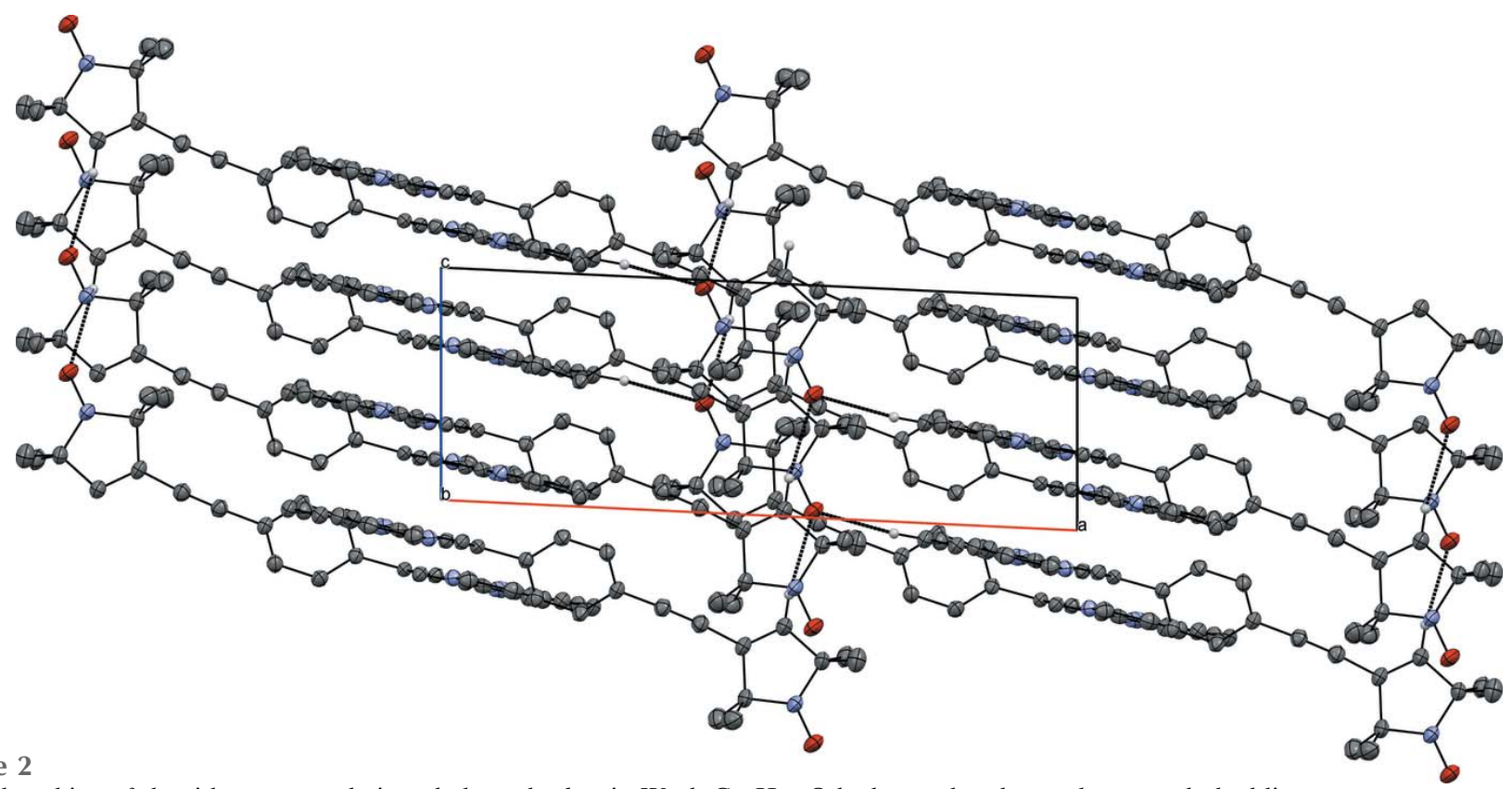

Figure 2

Crystal packing of the title compound viewed along the $b$ axis. Weak $\mathrm{C}-\mathrm{H} \cdots \mathrm{O}$ hydrogen bonds are shown as dashed lines

\section{Structural commentary}

The structure of the title compound (1) is shown in Fig. 1. The terpyridine group of (1) assumes an all-transoid conformation and is essentially planar with angles between the mean planes of the central pyridine (N1, C1-C5, r.m.s deviation from the mean plane $=0.006 \AA$ ) and the two outer rings amounting to $3.87(5)^{\circ}(\mathrm{N} 4, \mathrm{C} 27-\mathrm{C} 31$, r.m.s. deviation from the mean plane $=0.003 \AA$ ) and $1.98(5)^{\circ}(\mathrm{N} 2, \mathrm{C} 6-\mathrm{C} 10$, r.m.s deviation from the mean plane $=0.006 \AA$ ), respectively. The pyrroline- $N$-oxyl unit commonly found for such nitroxyls is seen in the structure and its mean plane $(\mathrm{N} 3, \mathrm{C} 19-\mathrm{C} 22$, r.m.s deviation from the mean plane $=0.006 \AA$ ) subtends a dihedral angle of $88.44(7)^{\circ}$ to the mean plane of the central pyridine ring (for similar structural motifs, see Margraf et al., 2009 and Schuetz et al., 2010). The subunits are linked by a 4-ethinylenephenylene group. The mean plane of the phenylene group (C11-C16, r.m.s deviation from the mean plane $<0.001 \AA$ ) is tilted with respect to both the central pyridine ring [dihedral angle of $51.36(5)^{\circ}$ ] and the pyrroline- $N$-oxyl [dihedral angle of $\left.37.62(7)^{\circ}\right]$. The angles $\mathrm{C} 18-\mathrm{C} 17-\mathrm{C} 14\left[177.35(19)^{\circ}\right]$ and
$\mathrm{C} 17-\mathrm{C} 18-\mathrm{C} 19\left[175.64(18)^{\circ}\right]$ are slightly lower than the $180^{\circ}$ expected for a strictly linear shape of the molecular backbone. Two short intramolecular hydrogen-nitrogen distances are observed between the two meta-protons of the central pyridine subunit and the nitrogen atoms of the external pyridine rings (Table 1). Murguly et al. (1999) propose weak intramolecular hydrogen bonds for these atoms. The intramolecular separation between the terpyridine group and the nitroxyl amounts to 14.120 (2) $\AA$ (measured between O1 and N1).

Table 1

Hydrogen-bond geometry $\left(\AA{ }^{\circ}\right)$.

$\mathrm{Cg}$ is the centroid of the C11-C16 ring.

\begin{tabular}{lllll}
\hline$D-\mathrm{H} \cdots A$ & $D-\mathrm{H}$ & $\mathrm{H} \cdots A$ & $D \cdots A$ & $D-\mathrm{H} \cdots A$ \\
\hline $\mathrm{C} 2-\mathrm{H} 2 \cdots \mathrm{N} 2$ & 0.95 & 2.50 & $2.815(2)$ & 99 \\
$\mathrm{C} 4-\mathrm{H} 4 \cdots \mathrm{N} 4$ & 0.95 & 2.46 & $2.778(2)$ & 100 \\
$\mathrm{C} 8-\mathrm{H} 8 \cdots \mathrm{O} 1^{\mathrm{i}}$ & 0.95 & 2.59 & $3.529(2)$ & 170 \\
$\mathrm{C} 16-\mathrm{H} 16 \cdots C g^{\mathrm{ii}}$ & 0.95 & 2.81 & $3.669(2)$ & 151 \\
$\mathrm{C} 22-\mathrm{H} 22 \cdots \mathrm{O} 1^{\mathrm{iii}}$ & 0.95 & 2.55 & $3.485(2)$ & 170 \\
\hline
\end{tabular}

Symmetry codes: (i) $x-1, y, z+1$; (ii) $x,-y+\frac{1}{2}, z+\frac{1}{2}$; (iii) $x, y, z+1$. 


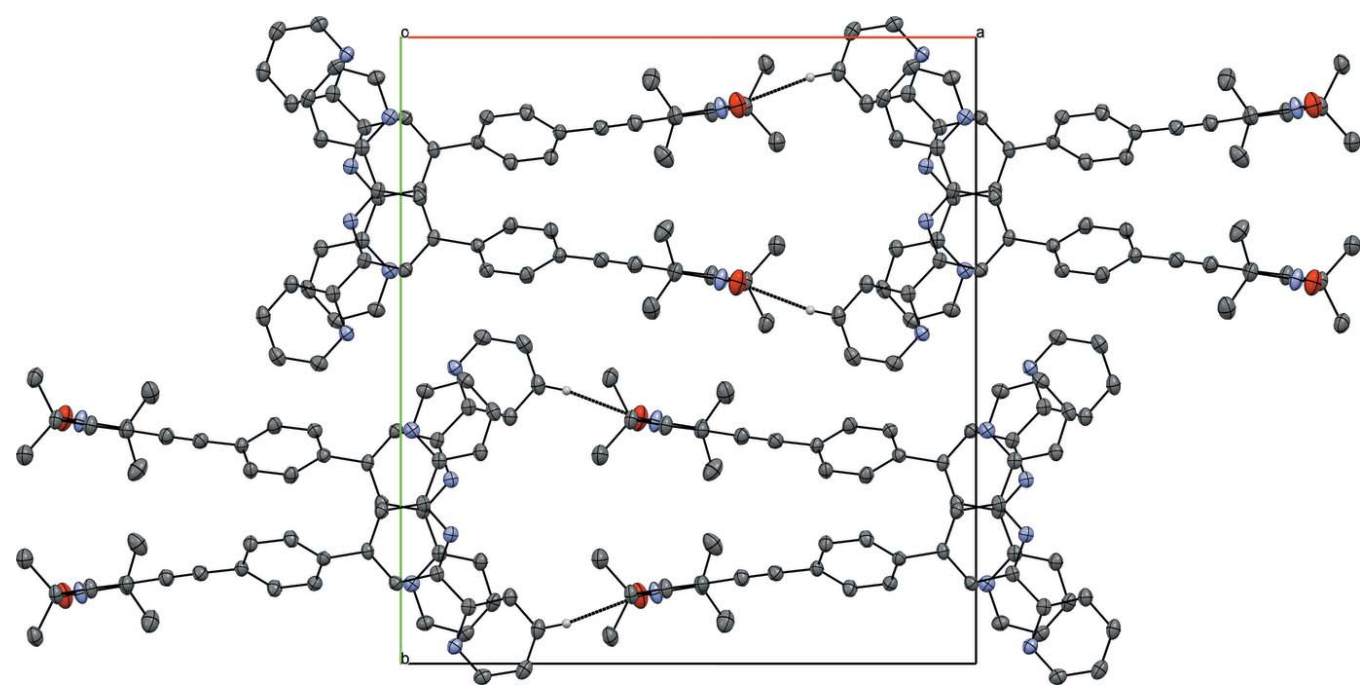

Figure 3

Crystal packing of the title compound viewed along the $c$ axis.

\section{Supramolecular features}

The packing within the crystal structure is shown in Figs. 2-4. The molecules are stacked in layers along [001] (Fig. 2.) The oxygen atom of the nitroxyl group forms weak hydrogen bonds to the protons of the para- $\mathrm{C}-\mathrm{H}$ group and the pyrroline $\mathrm{C}-\mathrm{H}$ group of neighbouring molecules (Table 1 ). These hydrogen bonds span a two-dimensional network within the (010) plane (Figs. 3 and 4 ). $\pi-\pi$ interactions are observed along [001] between the terpyridine subunits of neighbouring molecules (Figs. 3 and 5). These terpyridine subunits are arranged in a slipped face-to-face alignment (Janiak, 2000) with the shortest intermolecular distances between the pyridine rings amounting to 3.700 (1) $\AA$ (measured from the

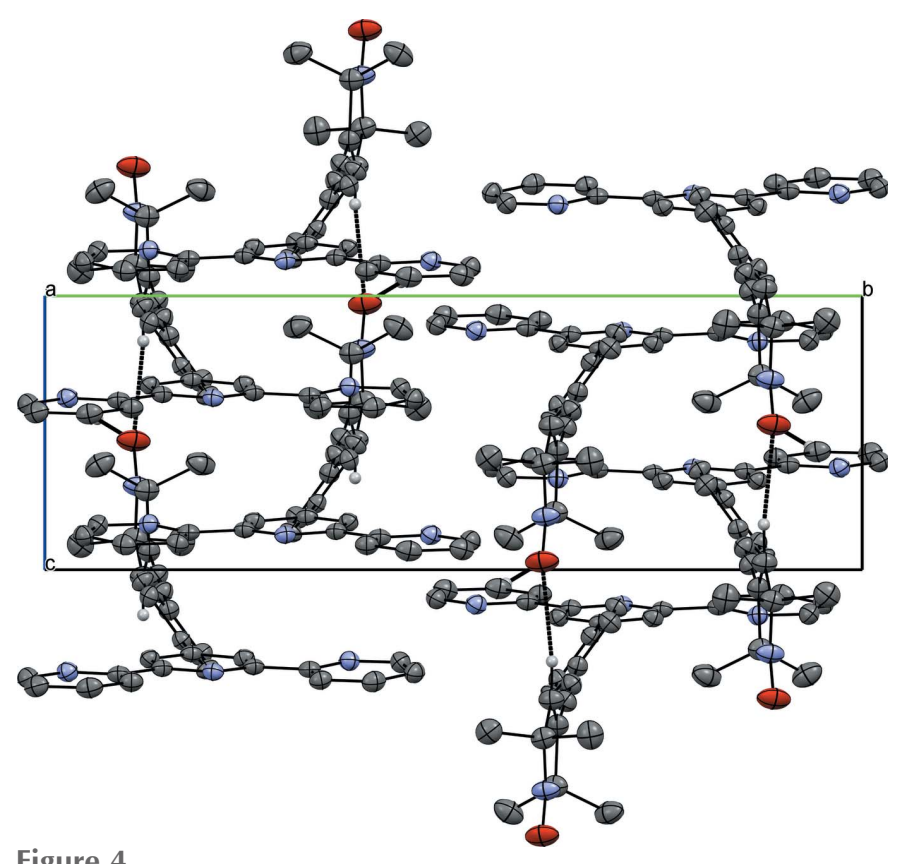

Figure 4

Crystal packing of the title compound viewed along the $a$ axis. centroid of N2, C6-C10 to the centroid of N4, C27-C31) and 3.781 (1) $\AA$ (centroid of N1, C1-C5 to the centroid of N4, C27-C31, see Fig. 5). Furthermore, the phenylene rings of neighbouring molecules show an edge-on $\mathrm{C}-\mathrm{H} \cdots \pi$ interaction along the same axis (Table 1 and Fig. 5). The nitroxyl groups are arranged in an alternating manner pointing in opposite directions. The shortest oxygen-oxygen separation between neighbouring molecules amounts to 5.412 (3) A. The oxygen-oxygen distance is an important factor determining the strength of through space exchange interactions of nitroxyls (Rajca et al. 2006).

\section{Database survey}

The Cambridge Structural Database (CSD, Version 5.36; Groom \& Allen, 2014) has been queried to find other terpyridine or 2,2,5,5-tetramethyl- $N$-oxyl-3-pyrroline derivatives. The terpyridine query revealed 3473 entries in the CSD

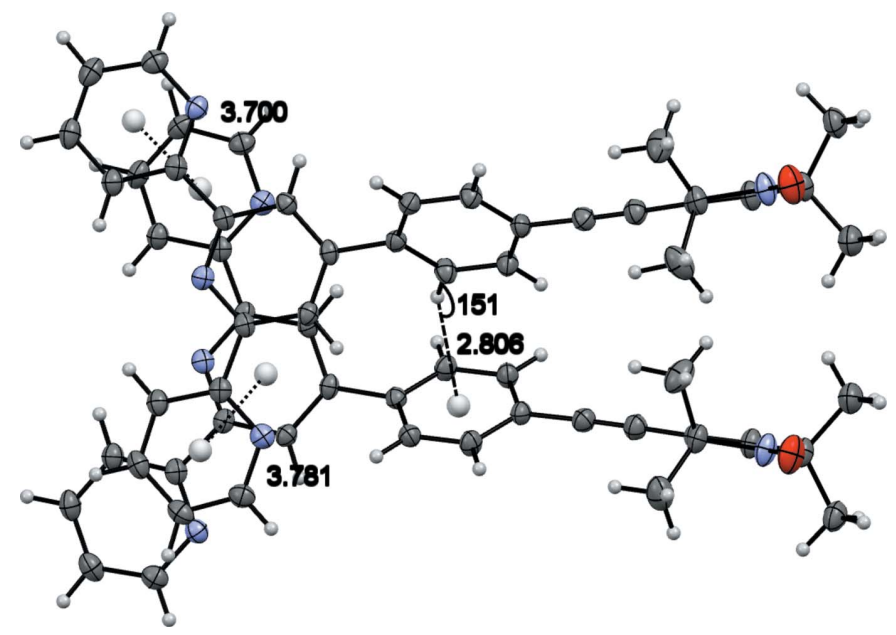

Figure 5

Closest distances between pyridine rings and edge-on $\mathrm{C}-\mathrm{H} \cdots \pi$ contact. 


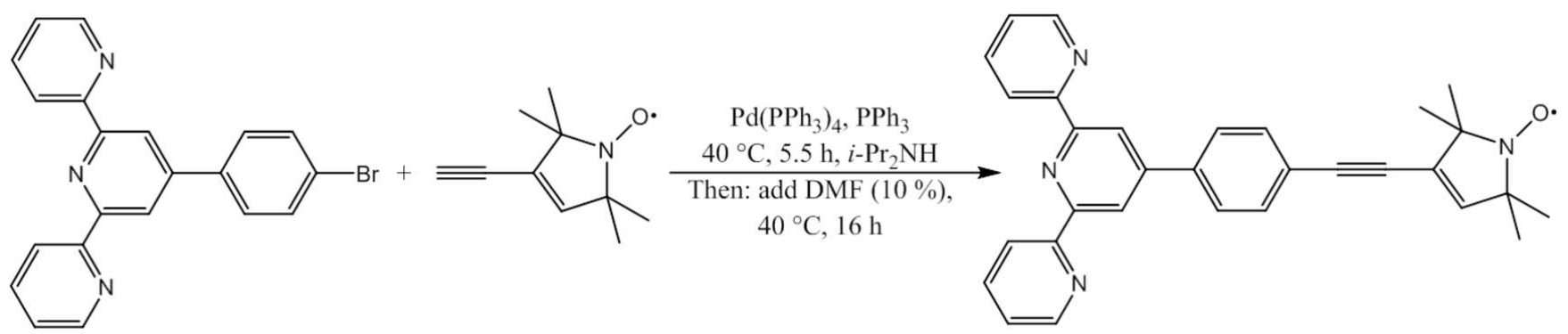

Figure 6

Scheme illustrating the synthesis of (1).

if metal complexes of terpyridine were included. For purely organic terpyridine compounds, the number of hits was reduced to 348 . Only 33 results for 2,2,5,5-tetramethyl- $N$-oxyl3 -pyrroline derivatives were found in the CSD. A combined query for structures which include both terpyridine and 2,2,5,5-tetramethyl- $N$-oxyl-3-pyrroline derivatives did not result in any hit. However, the authors are aware of at least one published crystal structure of a compound which contains both structural motifs (Ackermann et al., 2015).

\section{Synthesis and crystallization}

The title compound (1) is formed from 3-ethinyl-2,2,5,5tetramethyl-3-pyrroline- $N$-oxyl and $4^{\prime}$-(4-bromophenyl)$2,2^{\prime}: 6^{\prime}, 2^{\prime \prime}$-terpyridine using a Sonogashira-Hagihara crosscoupling reaction, as shown in Fig. $6.222 \mathrm{mg}(0.57 \mathrm{mmol})$ of $4^{\prime}$-(4-bromophenyl)-2,2':6', $2^{\prime \prime}$-terpyridine, $100 \mathrm{mg}(0.61 \mathrm{mmol})$

Table 2

Experimental details.

\begin{tabular}{|c|c|}
\hline \multicolumn{2}{|l|}{ Crystal data } \\
\hline Chemical formula & $\mathrm{C}_{31} \mathrm{H}_{27} \mathrm{~N}_{4} \mathrm{O}$ \\
\hline$M_{\mathrm{r}}$ & 471.56 \\
\hline Crystal system, space group & Monoclinic, $P 2_{1} / c$ \\
\hline Temperature $(\mathrm{K})$ & 123 \\
\hline$a, b, c(\AA)$ & $18.5666(8), 20.2009(9), 6.7749(2)$ \\
\hline$\beta\left(^{\circ}\right)$ & $92.743(3)$ \\
\hline$V\left(\mathrm{\AA}^{3}\right)$ & $2538.10(17)$ \\
\hline$Z$ & 4 \\
\hline Radiation type & Mo $K \alpha$ \\
\hline$\mu\left(\mathrm{mm}^{-1}\right)$ & 0.08 \\
\hline Crystal size $(\mathrm{mm})$ & $0.34 \times 0.12 \times 0.08$ \\
\hline \multicolumn{2}{|l|}{ Data collection } \\
\hline Diffractometer & Nonius KappaCCD \\
\hline Absorption correction & Multi-scan (Blessing, 1995) \\
\hline$T_{\min }, T_{\max }$ & $0.883,1.078$ \\
\hline $\begin{array}{l}\text { No. of measured, independent and } \\
\text { observed }[I>2 \sigma(I)] \text { reflections }\end{array}$ & $35758,6691,3221$ \\
\hline$R_{\text {int }}$ & 0.118 \\
\hline$(\sin \theta / \lambda)_{\max }\left(\AA^{-1}\right)$ & 0.685 \\
\hline \multicolumn{2}{|l|}{ Refinement } \\
\hline$R\left[F^{2}>2 \sigma\left(F^{2}\right)\right], w R\left(F^{2}\right), S$ & $0.049,0.122,0.89$ \\
\hline No. of reflections & 6691 \\
\hline No. of parameters & 329 \\
\hline $\mathrm{H}$-atom treatment & H-atom parameters constrained \\
\hline$\Delta \rho_{\max }, \Delta \rho_{\min }\left(\mathrm{e} \AA^{-3}\right)$ & $0.19,-0.23$ \\
\hline
\end{tabular}

Computer programs: DENZO and SCALEPACK (Otwinowski \& Minor, 1997), SHELXS97 (Sheldrick, 2008), SHELXL97 (Sheldrick, 2015) and OLEX2 (Dolomanov et al., 2009). of 3-ethinyl-2,2,5,5-tetramethyl-3-pyrroline- $N$-oxyl, $20 \mathrm{mg}$ $(0.076 \mathrm{mmol})$ of $\mathrm{PPh}_{3}$ and $40 \mathrm{mg}(0.035 \mathrm{mmol})$ of $\mathrm{Pd}\left(\mathrm{PPh}_{3}\right)_{4}$ were dissolved in $17 \mathrm{ml}$ of $i-\mathrm{Pr}_{2} \mathrm{NH}$ and stirred at $313 \mathrm{~K}$, yielding a yellow solution which turned orange over the course of $5 \mathrm{~min}$. Additionally, an orange precipitate was formed simultaneously. After $5.5 \mathrm{~h}, 2 \mathrm{ml}$ of dimethylformamide were added to the orange suspension. The stirring at $313 \mathrm{~K}$ was continued for $16 \mathrm{~h}$, after which time the solvents were removed under reduced pressure. The orange residues were suspended in a mixture of dichloromethane and cyclohexane (1:2) and subsequently subjected to column chromatography using aluminum oxide as stationary phase. A mixture of dichloromethane and cyclohexane was used as eluent. The volumetric ratio of both solvents was changed stepwise during the purification (from 1:8 to 8:1). The desired product was obtained in a yellow fraction and could be isolated by removing the eluents under reduced pressure (yield $80 \%$ ). The crystallization of (1) was achieved by slow evaporation of a solution of (1) in a 1:1 mixture of acetonitrile and dichloromethane. $4^{\prime}$-(4-Bromophenyl)-2,2' $: 6^{\prime}, 2^{\prime \prime}$-terpyridine was purchased from TCI Europe. 3-Ethinyl-2,2,5,5-tetramethyl-3pyrroline- $N$-oxyl was synthesized as described by Schiemann et al. (2007).

\section{Refinement}

Crystal data, data collection and structure refinement details are summarized in Table 2. All $\mathrm{H}$ atoms were fixed geometrically and allowed to ride on their parent $\mathrm{C}$ atoms, with $0.98 \AA$ with $U_{\text {iso }}(\mathrm{H})=1.5 U_{\text {eq }}(\mathrm{C})$ for methyl $\mathrm{H}$ atoms and $\mathrm{C}-\mathrm{H}$ $=0.95 \AA$ and $U_{\text {iso }}(\mathrm{H})=1.2 U_{\text {eq }}(\mathrm{C})$ for all other $\mathrm{H}$ atoms.

\section{Acknowledgements}

The authors thank Professor Dr A. C. Filippou for providing $\mathrm{X}$-ray infrastructure. OS thanks the DFG for funding via SFB 813.

\section{References}

Ackermann, K., Giannoulis, A., Cordes, D. B., Slawin, A. M. Z. \& Bode, B. E. (2015). Chem. Commun. 51, 5257-5260.

Bessel, C. A., See, R. F., Jameson, D. L., Churchill, M. R. \& Takeuchi, K. J. (1992). J. Chem. Soc. Dalton Trans. pp. 3223-3228.

Blessing, R. H. (1995). Acta Cryst. A51, 33-38. 
Constable, E. C., Baum, G., Bill, E., Dyson, R., van Eldik, R., Fenske, D., Kaderli, D., Morris, D., Neubrand, A., Neuburger, M., Smith, D. R., Wieghardt, K., Zehnder, M. \& Zuberbühler, A. D. (1999). Chem. Eur. J. 5, 498-508.

Dolomanov, O. V., Bourhis, L. J., Gildea, R. J., Howard, J. A. K. \& Puschmann, H. (2009). J. Appl. Cryst. 42, 339-341.

Eryazici, I., Moorefield, C. N., Durmus, S. \& Newkome, G. R. (2006). J. Org. Chem. 71, 1009-1014.

Fallahpour, R.-A., Neuburger, M. \& Zehnder, M. (1999). Polyhedron, 18, 2445-2454.

Folgado, J. V., Henke, W., Allmann, R., Stratemeier, H., BeltránPorter, D., Rojo, T. \& Reinen, D. (1990). Inorg. Chem. 29, 20352042.

Fritscher, J., Beyer, M. \& Schiemann, O. (2002). Chem. Phys. Lett. 364, 393-401.

Grave, C., Lentz, D., Schäfer, A., Samorì, P., Rabe, P. J., Franke, P. \& Schlüter, A. D. (2003). J. Am. Chem. Soc. 125, 6907-6918.

Groom, C. R. \& Allen, F. H. (2014). Angew. Chem. Int. Ed. 53, 662671.

Hogg, R. \& Wilkins, R. G. (1962). J. Chem. Soc. pp. 341-350.
Janiak, C. (2000). J. Chem. Soc. Dalton Trans. pp. 3885-3896.

Margraf, D., Schuetz, D., Prisner, T. F. \& Bats, J. W. (2009). Acta Cryst. E65, o1784.

Murguly, E., Norsten, T. B. \& Branda, N. (1999). J. Chem. Soc. Perkin Trans. 2, pp. 2789-2794.

Narr, E., Godt, A. \& Jeschke, G. (2002). Angew. Chem. Int. Ed. 41, 3907-3910.

Otwinowski, Z. \& Minor, W. (1997). Methods in Enzymology, Vol. 276, Macromolecular Crystallography, Part A, edited by C. W. Carter Jr \& R. M. Sweet, pp. 307-326. New York: Academic Press.

Rajca, A., Mukherjee, S., Pink, M. \& Rajca, S. (2006). J. Am. Chem. Soc. 128, 13497-13507.

Reginsson, G. W. \& Schiemann, O. (2011). Biochem. Soc. Trans. 39, 128-139.

Schiemann, O., Piton, N., Plackmeyer, J., Bode, B. E., Prisner, T. F. \& Engels, J. W. (2007). Nat. Protoc. 2, 904-923.

Schuetz, D., Margraf, D., Prisner, T. F. \& Bats, J. W. (2010). Acta Cryst. E66, 0729-o730.

Sheldrick, G. M. (2008). Acta Cryst. A64, 112-122.

Sheldrick, G. M. (2015). Acta Cryst. C71, 3-8. 


\section{supporting information}

Acta Cryst. (2015). E71, 870-874 [doi:10.1107/S2056989015012086]

\section{The crystal structure of $4^{\prime}-\{4-[(2,2,5,5$-tetramethyl- $N$-oxyl-3-pyrrolin-3- yl)ethynyl]phenyl\}-2,2':6',2"'-terpyridine}

\section{Andreas Meyer, Jennifer Wiecek, Gregor Schnakenburg and Olav Schiemann}

\section{Computing details}

Data collection: DENZO and SCALEPACK (Otwinowski \& Minor, 1997); cell refinement: SCALEPACK (Otwinowski \& Minor, 1997); data reduction: DENZO and SCALEPACK (Otwinowski \& Minor, 1997); program(s) used to solve structure: SHELXS97 (Sheldrick, 2008); program(s) used to refine structure: SHELXL97 (Sheldrick, 2015); molecular graphics: OLEX2 (Dolomanov et al., 2009); software used to prepare material for publication: OLEX2 (Dolomanov et al., 2009).

$\begin{array}{ll}\mathbf{4}^{\prime}-\left\{\mathbf{4}-\left[(\mathbf{2}, \mathbf{2 , 5}, \mathbf{5}-\text { Tetramethyl-N-oxyl-3-pyrrolin-3-yl)ethynyl]phenyl }\}-\mathbf{2}, \mathbf{2}^{\prime}: \mathbf{6}^{\prime}, \mathbf{2}^{\prime \prime} \text {-terpyridine }\right.\right. \\ \text { Crystal data } & \\ \mathrm{C}_{31} \mathrm{H}_{27} \mathrm{~N}_{4} \mathrm{O} & F(000)=996 \\ M_{r}=471.56 & D_{\mathrm{x}}=1.234 \mathrm{Mg} \mathrm{m}^{-3} \\ \text { Monoclinic, } P 2_{1} / c & \text { Mo } \mathrm{C} \alpha \text { radiation, } \lambda=0.71073 \AA \\ a=18.5666(8) \AA & \text { Cell parameters from } 9616 \text { reflections } \\ b=20.2009(9) \AA & \theta=1.0-29.1^{\circ} \\ c=6.7749(2) \AA & \mu=0.08 \mathrm{~mm}^{-1} \\ \beta=92.743(3)^{\circ} & T=123 \mathrm{~K} \\ V=2538.10(17) \AA^{3} & \text { Needle, clear yellow } \\ Z=4 & 0.34 \times 0.12 \times 0.08 \mathrm{~mm}\end{array}$

Data collection

Nonius KappaCCD diffractometer

Radiation source: sealed tube

Graphite monochromator Detector resolution: 8 pixels $\mathrm{mm}^{-1}$ fine slicing $\omega$ and $\varphi$ scans Absorption correction: multi-scan (Blessing, 1995)

$T_{\min }=0.883, T_{\max }=1.078$

\section{Refinement}

Refinement on $F^{2}$

Least-squares matrix: full

$R\left[F^{2}>2 \sigma\left(F^{2}\right)\right]=0.049$

$w R\left(F^{2}\right)=0.122$

$S=0.89$

6691 reflections

329 parameters

0 restraints
35758 measured reflections 6691 independent reflections 3221 reflections with $I>2 \sigma(I)$

$R_{\text {int }}=0.118$

$\theta_{\max }=29.2^{\circ}, \theta_{\min }=3.0^{\circ}$

$h=-25 \rightarrow 24$

$k=-24 \rightarrow 27$

$l=-9 \rightarrow 6$

Hydrogen site location: inferred from neighbouring sites

$\mathrm{H}$-atom parameters constrained

$w=1 /\left[\sigma^{2}\left(F_{\mathrm{o}}^{2}\right)+(0.052 P)^{2}\right]$

where $P=\left(F_{\mathrm{o}}^{2}+2 F_{\mathrm{c}}{ }^{2}\right) / 3$

$(\Delta / \sigma)_{\max }<0.001$

$\Delta \rho_{\max }=0.19 \mathrm{e} \AA^{-3}$

$\Delta \rho_{\min }=-0.23$ e $\AA^{-3}$ 


\section{Special details}

Geometry. All e.s.d.'s (except the e.s.d. in the dihedral angle between two 1.s. planes) are estimated using the full covariance matrix. The cell e.s.d.'s are taken into account individually in the estimation of e.s.d.'s in distances, angles and torsion angles; correlations between e.s.d.'s in cell parameters are only used when they are defined by crystal symmetry. An approximate (isotropic) treatment of cell e.s.d.'s is used for estimating e.s.d.'s involving l.s. planes.

Fractional atomic coordinates and isotropic or equivalent isotropic displacement parameters $\left(\AA^{2}\right)$

\begin{tabular}{|c|c|c|c|c|}
\hline & $x$ & $y$ & $z$ & $U_{\text {iso }} * / U_{\text {eq }}$ \\
\hline O1 & $0.58556(7)$ & $0.39166(8)$ & $0.02921(17)$ & 0.0449 (4) \\
\hline N1 & $-0.08706(7)$ & $0.29387(7)$ & $0.87301(18)$ & $0.0235(3)$ \\
\hline N2 & $-0.09424(7)$ & $0.47223(8)$ & $0.87295(19)$ & $0.0264(4)$ \\
\hline N3 & $0.55647(8)$ & $0.38695(8)$ & $0.1947(2)$ & $0.0332(4)$ \\
\hline N4 & $-0.01906(7)$ & $0.12743(7)$ & $0.83306(19)$ & $0.0262(3)$ \\
\hline $\mathrm{C} 1$ & $-0.06315(9)$ & $0.35653(9)$ & $0.8599(2)$ & $0.0221(4)$ \\
\hline $\mathrm{C} 2$ & $0.00840(9)$ & $0.37160(9)$ & $0.8258(2)$ & $0.0228(4)$ \\
\hline $\mathrm{H} 2$ & 0.0234 & 0.4164 & 0.8158 & $0.027 *$ \\
\hline C3 & $0.05751(9)$ & $0.32063(9)$ & 0.8067 (2) & $0.0224(4)$ \\
\hline C4 & $0.03323(9)$ & $0.25616(9)$ & $0.8239(2)$ & $0.0236(4)$ \\
\hline H4 & 0.0658 & 0.2202 & 0.8149 & $0.028 *$ \\
\hline $\mathrm{C} 5$ & $-0.03946(9)$ & $0.24445(9)$ & $0.8545(2)$ & $0.0223(4)$ \\
\hline C6 & $-0.11788(9)$ & $0.40962(9)$ & $0.8820(2)$ & $0.0244(4)$ \\
\hline $\mathrm{C} 7$ & $-0.19006(9)$ & $0.39411(9)$ & $0.9094(2)$ & $0.0280(4)$ \\
\hline H7 & -0.2054 & 0.3493 & 0.9130 & $0.034 *$ \\
\hline $\mathrm{C} 8$ & $-0.23880(10)$ & $0.44516(10)$ & $0.9310(2)$ & $0.0316(5)$ \\
\hline H8 & -0.2882 & 0.4359 & 0.9494 & $0.038^{*}$ \\
\hline C9 & $-0.21466(10)$ & $0.50989(10)$ & $0.9254(2)$ & $0.0319(5)$ \\
\hline H9 & -0.2467 & 0.5459 & 0.9426 & $0.038 *$ \\
\hline $\mathrm{C} 10$ & $-0.14220(10)$ & $0.52080(9)$ & $0.8941(2)$ & $0.0292(4)$ \\
\hline H10 & -0.1258 & 0.5653 & 0.8872 & $0.035 *$ \\
\hline $\mathrm{C} 11$ & $0.13389(9)$ & $0.33241(9)$ & $0.7586(2)$ & $0.0228(4)$ \\
\hline $\mathrm{C} 12$ & $0.14938(9)$ & $0.37194(9)$ & $0.5973(2)$ & $0.0260(4)$ \\
\hline H12 & 0.1113 & 0.3937 & 0.5245 & $0.031 *$ \\
\hline $\mathrm{C} 13$ & $0.21936(9)$ & $0.37982(9)$ & $0.5426(2)$ & $0.0273(4)$ \\
\hline H13 & 0.2290 & 0.4069 & 0.4323 & $0.033 *$ \\
\hline $\mathrm{C} 14$ & $0.27657(9)$ & $0.34827(9)$ & $0.6476(2)$ & $0.0244(4)$ \\
\hline $\mathrm{C} 15$ & $0.26114(9)$ & $0.30872(9)$ & $0.8088(2)$ & $0.0273(4)$ \\
\hline H15 & 0.2992 & 0.2870 & 0.8818 & $0.033 *$ \\
\hline $\mathrm{C} 16$ & $0.19076(9)$ & $0.30096(9)$ & $0.8632(2)$ & $0.0273(4)$ \\
\hline H16 & 0.1810 & 0.2738 & 0.9733 & $0.033 *$ \\
\hline $\mathrm{C} 17$ & $0.34837(10)$ & $0.35565(9)$ & 0.5825 (2) & 0.0275 (4) \\
\hline $\mathrm{C} 18$ & $0.40740(9)$ & $0.36297(9)$ & 0.5209 (2) & 0.0294 (4) \\
\hline $\mathrm{C} 19$ & $0.47510(9)$ & $0.37261(9)$ & $0.4332(2)$ & $0.0267(4)$ \\
\hline $\mathrm{C} 20$ & $0.47826(9)$ & $0.37532(10)$ & $0.2098(2)$ & 0.0294 (4) \\
\hline $\mathrm{C} 21$ & $0.59915(9)$ & $0.38953(10)$ & $0.3860(2)$ & 0.0305 (4) \\
\hline $\mathrm{C} 22$ & $0.53960(9)$ & $0.38011(10)$ & $0.5256(3)$ & 0.0308 (4) \\
\hline $\mathrm{H} 22$ & 0.5471 & 0.3796 & 0.6653 & $0.037 *$ \\
\hline $\mathrm{C} 23$ & $0.45763(11)$ & $0.30952(11)$ & $0.1131(3)$ & $0.0445(6)$ \\
\hline
\end{tabular}




\begin{tabular}{lllll} 
H23A & 0.4643 & 0.3122 & -0.0293 & $0.067^{*}$ \\
H23B & 0.4070 & 0.2997 & 0.1357 & $0.067^{*}$ \\
H23C & 0.4883 & 0.2743 & 0.1706 & $0.067^{*}$ \\
C24 & $0.43549(11)$ & $0.43246(11)$ & $0.1165(3)$ & $\left.0.0444^{*}\right)$ \\
H24A & 0.4511 & 0.4741 & 0.1791 & $0.067^{*}$ \\
H24B & 0.3840 & 0.4257 & 0.1354 & $0.067^{*}$ \\
H24C & 0.4438 & 0.4344 & -0.0252 & $0.067^{*}$ \\
C25 & $0.63565(10)$ & $0.45658(10)$ & $0.4093(3)$ & $0.0374(5)$ \\
H25A & 0.6686 & 0.4631 & 0.3023 & $0.056^{*}$ \\
H25B & 0.6628 & 0.4584 & 0.5368 & $0.056^{*}$ \\
H25C & 0.5990 & 0.4915 & 0.4039 & $0.056^{*}$ \\
C26 & $0.65362(10)$ & $0.33278(11)$ & $0.3968(3)$ & $0.0421(5)$ \\
H26A & 0.6280 & 0.2904 & 0.3849 & $0.063^{*}$ \\
H26B & 0.6812 & 0.3344 & 0.5236 & $0.063^{*}$ \\
H26C & 0.6866 & 0.3371 & 0.2887 & $0.063^{*}$ \\
C27 & $-0.06712(9)$ & $0.17596(9)$ & $0.8639(2)$ & $0.0230(4)$ \\
C28 & $-0.13860(9)$ & $0.16253(9)$ & $0.9022(2)$ & $0.0268(4)$ \\
H28 & -0.1714 & 0.1976 & 0.9238 & $0.032^{*}$ \\
C29 & $-0.16099(10)$ & $0.09759(9)$ & $0.9083(2)$ & $0.0295(4)$ \\
H29 & -0.2095 & 0.0873 & 0.9348 & $0.035^{*}$ \\
C30 & $-0.11244(10)$ & $0.04767(9)$ & $0.8756(2)$ & $0.0295(4)$ \\
H30 & -0.1268 & 0.0025 & 0.8789 & $0.035^{*}$ \\
C31 & $-0.04244(10)$ & $0.06489(9)$ & $0.8381(2)$ & $0.0290(4)$ \\
H31 & -0.0091 & 0.0304 & 0.8145 & $0.035^{*}$ \\
& & & & \\
\hline
\end{tabular}

Atomic displacement parameters $\left(\AA^{2}\right)$

\begin{tabular}{lllllll}
\hline & $U^{11}$ & $U^{22}$ & $U^{33}$ & $U^{12}$ & $U^{13}$ & $U^{23}$ \\
\hline O1 & $0.0353(8)$ & $0.0713(12)$ & $0.0294(7)$ & $-0.0087(7)$ & $0.0138(6)$ & $-0.0024(7)$ \\
N1 & $0.0240(8)$ & $0.0271(9)$ & $0.0196(7)$ & $0.0008(7)$ & $0.0026(6)$ & $-0.0010(6)$ \\
N2 & $0.0257(9)$ & $0.0283(10)$ & $0.0253(7)$ & $0.0025(7)$ & $0.0029(6)$ & $0.0004(6)$ \\
N3 & $0.0245(9)$ & $0.0507(12)$ & $0.0250(8)$ & $-0.0073(8)$ & $0.0075(6)$ & $-0.0021(7)$ \\
N4 & $0.0273(8)$ & $0.0282(10)$ & $0.0231(7)$ & $-0.0011(7)$ & $0.0018(6)$ & $-0.0003(6)$ \\
C1 & $0.0209(10)$ & $0.0277(11)$ & $0.0179(8)$ & $-0.0020(8)$ & $0.0016(6)$ & $0.0002(7)$ \\
C2 & $0.0224(9)$ & $0.0242(10)$ & $0.0219(8)$ & $-0.0020(8)$ & $0.0033(6)$ & $0.0002(7)$ \\
C3 & $0.0188(9)$ & $0.0301(11)$ & $0.0183(8)$ & $-0.0022(8)$ & $0.0025(6)$ & $0.0000(7)$ \\
C4 & $0.0218(10)$ & $0.0274(11)$ & $0.0221(8)$ & $0.0017(8)$ & $0.0043(7)$ & $0.0012(7)$ \\
C5 & $0.0218(9)$ & $0.0284(11)$ & $0.0171(7)$ & $-0.0018(8)$ & $0.0032(6)$ & $0.0008(7)$ \\
C6 & $0.0234(10)$ & $0.0314(11)$ & $0.0187(8)$ & $0.0007(8)$ & $0.0029(7)$ & $0.0003(7)$ \\
C7 & $0.0239(10)$ & $0.0345(12)$ & $0.0259(9)$ & $-0.0004(9)$ & $0.0041(7)$ & $0.0001(8)$ \\
C8 & $0.0225(10)$ & $0.0445(14)$ & $0.0282(9)$ & $0.0035(9)$ & $0.0055(7)$ & $0.0023(8)$ \\
C9 & $0.0287(11)$ & $0.0387(13)$ & $0.0285(9)$ & $0.0104(9)$ & $0.0040(7)$ & $0.0033(8)$ \\
C10 & $0.0329(11)$ & $0.0287(11)$ & $0.0261(9)$ & $0.0028(9)$ & $0.0018(7)$ & $0.0013(8)$ \\
C11 & $0.0207(9)$ & $0.0234(10)$ & $0.0243(8)$ & $-0.0003(8)$ & $0.0026(7)$ & $-0.0023(7)$ \\
C12 & $0.0234(10)$ & $0.0253(11)$ & $0.0293(9)$ & $0.0017(8)$ & $0.0020(7)$ & $0.0017(7)$ \\
C13 & $0.0241(10)$ & $0.0316(11)$ & $0.0267(9)$ & $-0.0001(8)$ & $0.0053(7)$ & $0.0062(8)$ \\
C14 & $0.0204(9)$ & $0.0255(11)$ & $0.0277(9)$ & $-0.0011(8)$ & $0.0060(7)$ & $-0.0007(7)$ \\
C15 & $0.0213(10)$ & $0.0310(11)$ & $0.0297(9)$ & $0.0007(8)$ & $0.0011(7)$ & $0.0040(8)$
\end{tabular}


supporting information

\begin{tabular}{lllllll} 
C16 & $0.0244(10)$ & $0.0314(11)$ & $0.0263(9)$ & $-0.0024(8)$ & $0.0035(7)$ & $0.0049(8)$ \\
C17 & $0.0261(11)$ & $0.0279(11)$ & $0.0288(9)$ & $-0.0010(8)$ & $0.0035(8)$ & $0.0025(7)$ \\
C18 & $0.0257(11)$ & $0.0320(12)$ & $0.0306(9)$ & $-0.0015(9)$ & $0.0031(8)$ & $0.0027(8)$ \\
C19 & $0.0216(10)$ & $0.0292(11)$ & $0.0302(9)$ & $-0.0006(8)$ & $0.0085(7)$ & $0.0005(8)$ \\
C20 & $0.0206(10)$ & $0.0379(12)$ & $0.0300(9)$ & $-0.0058(9)$ & $0.0042(7)$ & $0.0007(8)$ \\
C21 & $0.0208(10)$ & $0.0406(13)$ & $0.0304(9)$ & $-0.0031(9)$ & $0.0039(7)$ & $-0.0028(8)$ \\
C22 & $0.0236(10)$ & $0.0409(13)$ & $0.0281(9)$ & $-0.0031(9)$ & $0.0047(7)$ & $-0.0003(8)$ \\
C23 & $0.0447(13)$ & $0.0543(15)$ & $0.0347(11)$ & $-0.0176(11)$ & $0.0054(9)$ & $-0.0079(10)$ \\
C24 & $0.0350(12)$ & $0.0571(16)$ & $0.0414(11)$ & $0.0052(11)$ & $0.0050(9)$ & $0.0139(10)$ \\
C25 & $0.0267(11)$ & $0.0446(14)$ & $0.0416(11)$ & $-0.0060(9)$ & $0.0078(8)$ & $-0.0031(9)$ \\
C26 & $0.0297(11)$ & $0.0444(14)$ & $0.0526(13)$ & $0.0000(10)$ & $0.0070(9)$ & $0.0015(10)$ \\
C27 & $0.0229(10)$ & $0.0299(11)$ & $0.0164(8)$ & $0.0001(8)$ & $0.0006(6)$ & $0.0003(7)$ \\
C28 & $0.0234(10)$ & $0.0322(12)$ & $0.0248(9)$ & $-0.0010(9)$ & $0.0023(7)$ & $0.0017(8)$ \\
C29 & $0.0245(10)$ & $0.0365(12)$ & $0.0276(9)$ & $-0.0069(9)$ & $0.0024(7)$ & $0.0021(8)$ \\
C30 & $0.0336(11)$ & $0.0279(11)$ & $0.0270(9)$ & $-0.0070(9)$ & $0.0007(7)$ & $0.0022(8)$ \\
C31 & $0.0327(11)$ & $0.0270(11)$ & $0.0273(9)$ & $-0.0020(9)$ & $0.0011(7)$ & $-0.0019(8)$ \\
& & & & & & \\
\hline
\end{tabular}

Geometric parameters $\left(\AA,{ }^{\circ}\right)$

\begin{tabular}{llll}
\hline $\mathrm{O} 1-\mathrm{N} 3$ & $1.2712(17)$ & $\mathrm{C} 15-\mathrm{H} 15$ & 0.9500 \\
$\mathrm{~N} 1-\mathrm{C} 1$ & $1.346(2)$ & $\mathrm{C} 15-\mathrm{C} 16$ & $1.383(2)$ \\
$\mathrm{N} 1-\mathrm{C} 5$ & $1.343(2)$ & $\mathrm{C} 16-\mathrm{H} 16$ & 0.9500 \\
$\mathrm{~N} 2-\mathrm{C} 6$ & $1.341(2)$ & $\mathrm{C} 17-\mathrm{C} 18$ & $1.200(2)$ \\
$\mathrm{N} 2-\mathrm{C} 10$ & $1.337(2)$ & $\mathrm{C} 18-\mathrm{C} 19$ & $1.429(2)$ \\
$\mathrm{N} 3-\mathrm{C} 20$ & $1.479(2)$ & $\mathrm{C} 19-\mathrm{C} 20$ & $1.519(2)$ \\
$\mathrm{N} 3-\mathrm{C} 21$ & $1.487(2)$ & $\mathrm{C} 19-\mathrm{C} 22$ & $1.333(2)$ \\
$\mathrm{N} 4-\mathrm{C} 27$ & $1.349(2)$ & $\mathrm{C} 20-\mathrm{C} 23$ & $1.523(3)$ \\
$\mathrm{N} 4-\mathrm{C} 31$ & $1.337(2)$ & $\mathrm{C} 20-\mathrm{C} 24$ & $1.521(3)$ \\
$\mathrm{C} 1-\mathrm{C} 2$ & $1.393(2)$ & $\mathrm{C} 21-\mathrm{C} 22$ & $1.501(2)$ \\
$\mathrm{C} 1-\mathrm{C} 6$ & $1.490(2)$ & $\mathrm{C} 21-\mathrm{C} 25$ & $1.519(3)$ \\
$\mathrm{C} 2-\mathrm{H} 2$ & 0.9500 & $\mathrm{C} 21-\mathrm{C} 26$ & $1.528(3)$ \\
$\mathrm{C} 2-\mathrm{C} 3$ & $1.386(2)$ & $\mathrm{C} 22-\mathrm{H} 22$ & 0.9500 \\
$\mathrm{C} 3-\mathrm{C} 4$ & $1.385(2)$ & $\mathrm{C} 23-\mathrm{H} 23 \mathrm{~A}$ & 0.9800 \\
$\mathrm{C} 3-\mathrm{C} 11$ & $1.489(2)$ & $\mathrm{C} 23-\mathrm{H} 23 \mathrm{~B}$ & 0.9800 \\
$\mathrm{C} 4-\mathrm{H} 4$ & 0.9500 & $\mathrm{C} 23-\mathrm{H} 23 \mathrm{C}$ & 0.9800 \\
$\mathrm{C} 4-\mathrm{C} 5$ & $1.396(2)$ & $\mathrm{C} 24-\mathrm{H} 24 \mathrm{~A}$ & 0.9800 \\
$\mathrm{C} 5-\mathrm{C} 27$ & $1.478(2)$ & $\mathrm{C} 24-\mathrm{H} 24 \mathrm{~B}$ & 0.9800 \\
$\mathrm{C} 6-\mathrm{C} 7$ & $1.397(2)$ & $\mathrm{C} 24-\mathrm{H} 24 \mathrm{C}$ & 0.9800 \\
$\mathrm{C} 7-\mathrm{H} 7$ & $\mathrm{C} 25-\mathrm{H} 25 \mathrm{~A}$ & 0.9800 \\
$\mathrm{C} 7-\mathrm{C} 8$ & 1.9500 & $\mathrm{C} 25-\mathrm{H} 25 \mathrm{~B}$ & 0.9800 \\
$\mathrm{C} 8-\mathrm{H} 8$ & $\mathrm{C} 25-\mathrm{H} 25 \mathrm{C}$ & 0.9800 \\
$\mathrm{C} 8-\mathrm{C} 9$ & $\mathrm{C} 26-\mathrm{H} 26 \mathrm{~A}$ & 0.9800 \\
$\mathrm{C} 9-\mathrm{H} 9$ & $\mathrm{C} 26-\mathrm{H} 26 \mathrm{~B}$ & 0.9800 \\
$\mathrm{C} 9-\mathrm{C} 10$ & $1.384(2)$ & $\mathrm{C} 26-\mathrm{H} 26 \mathrm{C}$ & 0.9800 \\
$\mathrm{C} 10-\mathrm{H} 10$ & $\mathrm{C} 27-\mathrm{C} 28$ & $1.391(2)$ \\
$\mathrm{C} 11-\mathrm{C} 12$ & $\mathrm{C} 28-\mathrm{H} 28$ & 0.9500 \\
$\mathrm{C} 11-\mathrm{C} 16$ & $\mathrm{C} 28-\mathrm{C} 29$ & 0.9500 \\
$\mathrm{C} 12-\mathrm{H} 12$ & $\mathrm{C} 29-\mathrm{H} 29$ &
\end{tabular}




$$
\begin{aligned}
& \text { C12-C13 } \\
& \text { C13-H13 } \\
& \text { C13-C14 } \\
& \text { C14-C15 } \\
& \text { C14-C17 }
\end{aligned}
$$$$
\text { C5-N1-C1 }
$$$$
\mathrm{C} 10-\mathrm{N} 2-\mathrm{C} 6
$$$$
\mathrm{O} 1-\mathrm{N} 3-\mathrm{C} 20
$$$$
\mathrm{O} 1-\mathrm{N} 3-\mathrm{C} 21
$$$$
\mathrm{C} 20-\mathrm{N} 3-\mathrm{C} 21
$$$$
\mathrm{C} 31-\mathrm{N} 4-\mathrm{C} 27
$$$$
\mathrm{N} 1-\mathrm{C} 1-\mathrm{C} 2
$$$$
\mathrm{N} 1-\mathrm{C} 1-\mathrm{C} 6
$$$$
\mathrm{C} 2-\mathrm{C} 1-\mathrm{C} 6
$$$$
\mathrm{C} 1-\mathrm{C} 2-\mathrm{H} 2
$$$$
\mathrm{C} 3-\mathrm{C} 2-\mathrm{C} 1
$$$$
\mathrm{C} 3-\mathrm{C} 2-\mathrm{H} 2
$$$$
\mathrm{C} 2-\mathrm{C} 3-\mathrm{C} 11
$$$$
\mathrm{C} 4-\mathrm{C} 3-\mathrm{C} 2
$$$$
\mathrm{C} 4-\mathrm{C} 3-\mathrm{C} 11
$$$$
\mathrm{C} 3-\mathrm{C} 4-\mathrm{H} 4
$$$$
\mathrm{C} 3-\mathrm{C} 4-\mathrm{C} 5
$$$$
\mathrm{C} 5-\mathrm{C} 4-\mathrm{H} 4
$$$$
\mathrm{N} 1-\mathrm{C} 5-\mathrm{C} 4
$$$$
\mathrm{N} 1-\mathrm{C} 5-\mathrm{C} 27
$$$$
\mathrm{C} 4-\mathrm{C} 5-\mathrm{C} 27
$$$$
\mathrm{N} 2-\mathrm{C} 6-\mathrm{C} 1
$$$$
\text { N2-C6-C7 }
$$$$
\mathrm{C} 7-\mathrm{C} 6-\mathrm{C} 1
$$$$
\text { C6- } 7 \text { 7- } \mathrm{H} 7
$$$$
\mathrm{C} 8-\mathrm{C} 7-\mathrm{C} 6
$$$$
\mathrm{C} 8-\mathrm{C} 7-\mathrm{H} 7
$$$$
\mathrm{C} 7-\mathrm{C} 8-\mathrm{H} 8
$$$$
\mathrm{C} 9-\mathrm{C} 8-\mathrm{C} 7
$$$$
\mathrm{C} 9-\mathrm{C} 8-\mathrm{H} 8
$$$$
\mathrm{C} 8-\mathrm{C} 9-\mathrm{H} 9
$$$$
\text { C } 8-\mathrm{C} 9-\mathrm{C} 10
$$$$
\mathrm{C} 10-\mathrm{C} 9-\mathrm{H} 9
$$$$
\mathrm{N} 2-\mathrm{C} 10-\mathrm{C} 9
$$$$
\mathrm{N} 2-\mathrm{C} 10-\mathrm{H} 10
$$$$
\text { C9- } 110-\mathrm{H} 10
$$$$
\mathrm{C} 12-\mathrm{C} 11-\mathrm{C} 3
$$$$
\mathrm{C} 12-\mathrm{C} 11-\mathrm{C} 16
$$$$
\mathrm{C} 16-\mathrm{C} 11-\mathrm{C} 3
$$$$
\mathrm{C} 11-\mathrm{C} 12-\mathrm{H} 12
$$$$
\mathrm{C} 13-\mathrm{C} 12-\mathrm{C} 11
$$$$
\mathrm{C} 13-\mathrm{C} 12-\mathrm{H} 12
$$

1.377 (2)

0.9500

$1.403(2)$

$1.394(2)$

$1.432(2)$

$118.19(14)$

$117.76(15)$

$122.18(13)$

$122.33(13)$

$115.43(12)$

$117.70(15)$

$122.47(16)$

$116.22(15)$

$121.31(16)$

120.3

119.35 (16)

120.3

$122.65(16)$

$118.22(15)$

$119.06(15)$

120.2

119.52 (17)

120.2

$122.23(16)$

$117.40(15)$

$120.36(16)$

$116.62(15)$

$122.39(16)$

120.99 (17)

120.6

118.88 (18)

120.6

120.4

119.14 (17)

120.4

120.9

118.13 (17)

120.9

$123.68(18)$

118.2

118.2

$119.82(15)$

$118.62(15)$

$121.41(15)$

119.7

120.64 (16)

119.7

$$
\begin{aligned}
& \mathrm{C} 29-\mathrm{C} 30 \\
& \mathrm{C} 30-\mathrm{H} 30 \\
& \mathrm{C} 30-\mathrm{C} 31 \\
& \mathrm{C} 31-\mathrm{H} 31
\end{aligned}
$$

$\mathrm{C} 22-\mathrm{C} 19-\mathrm{C} 18$

$\mathrm{C} 22-\mathrm{C} 19-\mathrm{C} 20$

N3-C20-C19

$\mathrm{N} 3-\mathrm{C} 20-\mathrm{C} 23$

N3- C20-C24

$\mathrm{C} 19-\mathrm{C} 20-\mathrm{C} 23$

$\mathrm{C} 19-\mathrm{C} 20-\mathrm{C} 24$

$\mathrm{C} 24-\mathrm{C} 20-\mathrm{C} 23$

N3-C21-C22

N3-C21-C25

$\mathrm{N} 3-\mathrm{C} 21-\mathrm{C} 26$

$\mathrm{C} 22-\mathrm{C} 21-\mathrm{C} 25$

$\mathrm{C} 22-\mathrm{C} 21-\mathrm{C} 26$

$\mathrm{C} 25-\mathrm{C} 21-\mathrm{C} 26$

$\mathrm{C} 19-\mathrm{C} 22-\mathrm{C} 21$

$\mathrm{C} 19-\mathrm{C} 22-\mathrm{H} 22$

$\mathrm{C} 21-\mathrm{C} 22-\mathrm{H} 22$

$\mathrm{C} 20-\mathrm{C} 23-\mathrm{H} 23 \mathrm{~A}$

$\mathrm{C} 20-\mathrm{C} 23-\mathrm{H} 23 \mathrm{~B}$

$\mathrm{C} 20-\mathrm{C} 23-\mathrm{H} 23 \mathrm{C}$

$\mathrm{H} 23 \mathrm{~A}-\mathrm{C} 23-\mathrm{H} 23 \mathrm{~B}$

$\mathrm{H} 23 \mathrm{~A}-\mathrm{C} 23-\mathrm{H} 23 \mathrm{C}$

$\mathrm{H} 23 \mathrm{~B}-\mathrm{C} 23-\mathrm{H} 23 \mathrm{C}$

$\mathrm{C} 20-\mathrm{C} 24-\mathrm{H} 24 \mathrm{~A}$

C20-C24-H24B

$\mathrm{C} 20-\mathrm{C} 24-\mathrm{H} 24 \mathrm{C}$

$\mathrm{H} 24 \mathrm{~A}-\mathrm{C} 24-\mathrm{H} 24 \mathrm{~B}$

$\mathrm{H} 24 \mathrm{~A}-\mathrm{C} 24-\mathrm{H} 24 \mathrm{C}$

$\mathrm{H} 24 \mathrm{~B}-\mathrm{C} 24-\mathrm{H} 24 \mathrm{C}$

$\mathrm{C} 21-\mathrm{C} 25-\mathrm{H} 25 \mathrm{~A}$

$\mathrm{C} 21-\mathrm{C} 25-\mathrm{H} 25 \mathrm{~B}$

$\mathrm{C} 21-\mathrm{C} 25-\mathrm{H} 25 \mathrm{C}$

$\mathrm{H} 25 \mathrm{~A}-\mathrm{C} 25-\mathrm{H} 25 \mathrm{~B}$

$\mathrm{H} 25 \mathrm{~A}-\mathrm{C} 25-\mathrm{H} 25 \mathrm{C}$

$\mathrm{H} 25 \mathrm{~B}-\mathrm{C} 25-\mathrm{H} 25 \mathrm{C}$

$\mathrm{C} 21-\mathrm{C} 26-\mathrm{H} 26 \mathrm{~A}$

C21-C26-H26B

$\mathrm{C} 21-\mathrm{C} 26-\mathrm{H} 26 \mathrm{C}$

$\mathrm{H} 26 \mathrm{~A}-\mathrm{C} 26-\mathrm{H} 26 \mathrm{~B}$

$\mathrm{H} 26 \mathrm{~A}-\mathrm{C} 26-\mathrm{H} 26 \mathrm{C}$

$\mathrm{H} 26 \mathrm{~B}-\mathrm{C} 26-\mathrm{H} 26 \mathrm{C}$

$\mathrm{N} 4-\mathrm{C} 27-\mathrm{C} 5$
1.377 (3)

0.9500

$1.381(2)$

0.9500

$127.46(16)$

$112.80(15)$

$99.16(13)$

$109.66(15)$

$110.21(15)$

$112.11(16)$

$113.40(16)$

$111.60(16)$

$99.62(13)$

$109.78(15)$

$109.83(15)$

$112.68(15)$

$112.36(16)$

$111.89(15)$

$112.98(15)$

123.5

123.5

109.5

109.5

109.5

109.5

109.5

109.5

109.5

109.5

109.5

109.5

109.5

109.5

109.5

109.5

109.5

109.5

109.5

109.5

109.5

109.5

109.5

109.5

109.5

109.5

116.10 (15) 


\begin{tabular}{|c|c|c|c|}
\hline $\mathrm{C} 12-\mathrm{C} 13-\mathrm{H} 13$ & 119.6 & $\mathrm{~N} 4-\mathrm{C} 27-\mathrm{C} 28$ & $122.10(17)$ \\
\hline $\mathrm{C} 12-\mathrm{C} 13-\mathrm{C} 14$ & $120.79(16)$ & $\mathrm{C} 28-\mathrm{C} 27-\mathrm{C} 5$ & $121.80(16)$ \\
\hline $\mathrm{C} 14-\mathrm{C} 13-\mathrm{H} 13$ & 119.6 & $\mathrm{C} 27-\mathrm{C} 28-\mathrm{H} 28$ & 120.5 \\
\hline $\mathrm{C} 13-\mathrm{C} 14-\mathrm{C} 17$ & $119.32(15)$ & $\mathrm{C} 29-\mathrm{C} 28-\mathrm{C} 27$ & $118.90(17)$ \\
\hline $\mathrm{C} 15-\mathrm{C} 14-\mathrm{C} 13$ & $118.61(15)$ & $\mathrm{C} 29-\mathrm{C} 28-\mathrm{H} 28$ & 120.5 \\
\hline $\mathrm{C} 15-\mathrm{C} 14-\mathrm{C} 17$ & $122.03(16)$ & $\mathrm{C} 28-\mathrm{C} 29-\mathrm{H} 29$ & 120.3 \\
\hline $\mathrm{C} 14-\mathrm{C} 15-\mathrm{H} 15$ & 119.8 & $\mathrm{C} 28-\mathrm{C} 29-\mathrm{C} 30$ & $119.45(17)$ \\
\hline $\mathrm{C} 16-\mathrm{C} 15-\mathrm{C} 14$ & $120.38(16)$ & $\mathrm{C} 30-\mathrm{C} 29-\mathrm{H} 29$ & 120.3 \\
\hline $\mathrm{C} 16-\mathrm{C} 15-\mathrm{H} 15$ & 119.8 & $\mathrm{C} 29-\mathrm{C} 30-\mathrm{H} 30$ & 120.9 \\
\hline $\mathrm{C} 11-\mathrm{C} 16-\mathrm{H} 16$ & 119.5 & $\mathrm{C} 29-\mathrm{C} 30-\mathrm{C} 31$ & $118.29(18)$ \\
\hline $\mathrm{C} 15-\mathrm{C} 16-\mathrm{C} 11$ & $120.96(16)$ & $\mathrm{C} 31-\mathrm{C} 30-\mathrm{H} 30$ & 120.9 \\
\hline $\mathrm{C} 15-\mathrm{C} 16-\mathrm{H} 16$ & 119.5 & $\mathrm{~N} 4-\mathrm{C} 31-\mathrm{C} 30$ & $123.55(17)$ \\
\hline $\mathrm{C} 18-\mathrm{C} 17-\mathrm{C} 14$ & $177.35(19)$ & $\mathrm{N} 4-\mathrm{C} 31-\mathrm{H} 31$ & 118.2 \\
\hline $\mathrm{C} 17-\mathrm{C} 18-\mathrm{C} 19$ & $175.64(18)$ & $\mathrm{C} 30-\mathrm{C} 31-\mathrm{H} 31$ & 118.2 \\
\hline $\mathrm{C} 18-\mathrm{C} 19-\mathrm{C} 20$ & $119.74(15)$ & & \\
\hline $\mathrm{O} 1-\mathrm{N} 3-\mathrm{C} 20-\mathrm{C} 19$ & $178.70(16)$ & $\mathrm{C} 6-\mathrm{C} 7-\mathrm{C} 8-\mathrm{C} 9$ & $-0.2(2)$ \\
\hline $\mathrm{O} 1-\mathrm{N} 3-\mathrm{C} 20-\mathrm{C} 23$ & $61.1(2)$ & $\mathrm{C} 7-\mathrm{C} 8-\mathrm{C} 9-\mathrm{C} 10$ & $1.3(2)$ \\
\hline $\mathrm{O} 1-\mathrm{N} 3-\mathrm{C} 20-\mathrm{C} 24$ & $-62.1(2)$ & $\mathrm{C} 8-\mathrm{C} 9-\mathrm{C} 10-\mathrm{N} 2$ & $-1.4(2)$ \\
\hline $\mathrm{O} 1-\mathrm{N} 3-\mathrm{C} 21-\mathrm{C} 22$ & $-178.63(17)$ & $\mathrm{C} 10-\mathrm{N} 2-\mathrm{C} 6-\mathrm{C} 1$ & $-179.46(13)$ \\
\hline $\mathrm{O} 1-\mathrm{N} 3-\mathrm{C} 21-\mathrm{C} 25$ & $62.9(2)$ & $\mathrm{C} 10-\mathrm{N} 2-\mathrm{C} 6-\mathrm{C} 7$ & $1.0(2)$ \\
\hline $\mathrm{O} 1-\mathrm{N} 3-\mathrm{C} 21-\mathrm{C} 26$ & $-60.5(2)$ & $\mathrm{C} 11-\mathrm{C} 3-\mathrm{C} 4-\mathrm{C} 5$ & $-175.31(13)$ \\
\hline $\mathrm{N} 1-\mathrm{C} 1-\mathrm{C} 2-\mathrm{C} 3$ & $-0.7(2)$ & $\mathrm{C} 11-\mathrm{C} 12-\mathrm{C} 13-\mathrm{C} 14$ & $0.0(3)$ \\
\hline $\mathrm{N} 1-\mathrm{C} 1-\mathrm{C} 6-\mathrm{N} 2$ & $178.75(13)$ & $\mathrm{C} 12-\mathrm{C} 11-\mathrm{C} 16-\mathrm{C} 15$ & $0.1(3)$ \\
\hline $\mathrm{N} 1-\mathrm{C} 1-\mathrm{C} 6-\mathrm{C} 7$ & $-1.7(2)$ & $\mathrm{C} 12-\mathrm{C} 13-\mathrm{C} 14-\mathrm{C} 15$ & $0.0(3)$ \\
\hline $\mathrm{N} 1-\mathrm{C} 5-\mathrm{C} 27-\mathrm{N} 4$ & $176.14(13)$ & $\mathrm{C} 12-\mathrm{C} 13-\mathrm{C} 14-\mathrm{C} 17$ & $177.81(16)$ \\
\hline $\mathrm{N} 1-\mathrm{C} 5-\mathrm{C} 27-\mathrm{C} 28$ & $-3.8(2)$ & $\mathrm{C} 13-\mathrm{C} 14-\mathrm{C} 15-\mathrm{C} 16$ & $0.0(3)$ \\
\hline $\mathrm{N} 2-\mathrm{C} 6-\mathrm{C} 7-\mathrm{C} 8$ & $-1.0(2)$ & $\mathrm{C} 14-\mathrm{C} 15-\mathrm{C} 16-\mathrm{C} 11$ & $-0.1(3)$ \\
\hline $\mathrm{N} 3-\mathrm{C} 21-\mathrm{C} 22-\mathrm{C} 19$ & $0.7(2)$ & $\mathrm{C} 16-\mathrm{C} 11-\mathrm{C} 12-\mathrm{C} 13$ & $-0.1(3)$ \\
\hline $\mathrm{N} 4-\mathrm{C} 27-\mathrm{C} 28-\mathrm{C} 29$ & $-0.1(2)$ & $\mathrm{C} 17-\mathrm{C} 14-\mathrm{C} 15-\mathrm{C} 16$ & $-177.74(17)$ \\
\hline $\mathrm{C} 1-\mathrm{N} 1-\mathrm{C} 5-\mathrm{C} 4$ & $0.5(2)$ & $\mathrm{C} 18-\mathrm{C} 19-\mathrm{C} 20-\mathrm{N} 3$ & $178.91(16)$ \\
\hline $\mathrm{C} 1-\mathrm{N} 1-\mathrm{C} 5-\mathrm{C} 27$ & $-178.37(13)$ & $\mathrm{C} 18-\mathrm{C} 19-\mathrm{C} 20-\mathrm{C} 23$ & $-65.4(2)$ \\
\hline $\mathrm{C} 1-\mathrm{C} 2-\mathrm{C} 3-\mathrm{C} 4$ & $-0.5(2)$ & $\mathrm{C} 18-\mathrm{C} 19-\mathrm{C} 20-\mathrm{C} 24$ & $62.1(2)$ \\
\hline $\mathrm{C} 1-\mathrm{C} 2-\mathrm{C} 3-\mathrm{C} 11$ & $176.34(14)$ & $\mathrm{C} 18-\mathrm{C} 19-\mathrm{C} 22-\mathrm{C} 21$ & $-179.71(19)$ \\
\hline $\mathrm{C} 1-\mathrm{C} 6-\mathrm{C} 7-\mathrm{C} 8$ & $179.43(14)$ & $\mathrm{C} 20-\mathrm{N} 3-\mathrm{C} 21-\mathrm{C} 22$ & $-1.4(2)$ \\
\hline $\mathrm{C} 2-\mathrm{C} 1-\mathrm{C} 6-\mathrm{N} 2$ & $-1.8(2)$ & $\mathrm{C} 20-\mathrm{N} 3-\mathrm{C} 21-\mathrm{C} 25$ & $-119.89(17)$ \\
\hline $\mathrm{C} 2-\mathrm{C} 1-\mathrm{C} 6-\mathrm{C} 7$ & $177.78(15)$ & $\mathrm{C} 20-\mathrm{N} 3-\mathrm{C} 21-\mathrm{C} 26$ & $116.68(17)$ \\
\hline $\mathrm{C} 2-\mathrm{C} 3-\mathrm{C} 4-\mathrm{C} 5$ & $1.6(2)$ & $\mathrm{C} 20-\mathrm{C} 19-\mathrm{C} 22-\mathrm{C} 21$ & $0.1(2)$ \\
\hline $\mathrm{C} 2-\mathrm{C} 3-\mathrm{C} 11-\mathrm{C} 12$ & $-51.0(2)$ & $\mathrm{C} 21-\mathrm{N} 3-\mathrm{C} 20-\mathrm{C} 19$ & $1.5(2)$ \\
\hline $\mathrm{C} 2-\mathrm{C} 3-\mathrm{C} 11-\mathrm{C} 16$ & $133.51(18)$ & $\mathrm{C} 21-\mathrm{N} 3-\mathrm{C} 20-\mathrm{C} 23$ & $-116.05(17)$ \\
\hline $\mathrm{C} 3-\mathrm{C} 4-\mathrm{C} 5-\mathrm{N} 1$ & $-1.7(2)$ & $\mathrm{C} 21-\mathrm{N} 3-\mathrm{C} 20-\mathrm{C} 24$ & $120.71(17)$ \\
\hline $\mathrm{C} 3-\mathrm{C} 4-\mathrm{C} 5-\mathrm{C} 27$ & $177.14(13)$ & $\mathrm{C} 22-\mathrm{C} 19-\mathrm{C} 20-\mathrm{N} 3$ & $-1.0(2)$ \\
\hline $\mathrm{C} 3-\mathrm{C} 11-\mathrm{C} 12-\mathrm{C} 13$ & $-175.65(16)$ & $\mathrm{C} 22-\mathrm{C} 19-\mathrm{C} 20-\mathrm{C} 23$ & $114.73(18)$ \\
\hline $\mathrm{C} 3-\mathrm{C} 11-\mathrm{C} 16-\mathrm{C} 15$ & $175.58(16)$ & $\mathrm{C} 22-\mathrm{C} 19-\mathrm{C} 20-\mathrm{C} 24$ & $-117.78(18)$ \\
\hline $\mathrm{C} 4-\mathrm{C} 3-\mathrm{C} 11-\mathrm{C} 12$ & $125.74(17)$ & $\mathrm{C} 25-\mathrm{C} 21-\mathrm{C} 22-\mathrm{C} 19$ & $117.02(18)$ \\
\hline $\mathrm{C} 4-\mathrm{C} 3-\mathrm{C} 11-\mathrm{C} 16$ & $-49.7(2)$ & $\mathrm{C} 26-\mathrm{C} 21-\mathrm{C} 22-\mathrm{C} 19$ & $-115.47(18)$ \\
\hline $\mathrm{C} 4-\mathrm{C} 5-\mathrm{C} 27-\mathrm{N} 4$ & $-2.7(2)$ & $\mathrm{C} 27-\mathrm{N} 4-\mathrm{C} 31-\mathrm{C} 30$ & $-0.9(2)$ \\
\hline $\mathrm{C} 4-\mathrm{C} 5-\mathrm{C} 27-\mathrm{C} 28$ & $177.36(14)$ & $\mathrm{C} 27-\mathrm{C} 28-\mathrm{C} 29-\mathrm{C} 30$ & $-0.3(2)$ \\
\hline
\end{tabular}




\section{supporting information}

$\begin{array}{llll}\mathrm{C} 5-\mathrm{N} 1-\mathrm{C} 1-\mathrm{C} 2 & 0.7(2) & \mathrm{C} 28-\mathrm{C} 29-\mathrm{C} 30-\mathrm{C} 31 & 0.1(2) \\ \mathrm{C} 5-\mathrm{N} 1-\mathrm{C} 1-\mathrm{C} 6 & -179.79(13) & \mathrm{C} 29-\mathrm{C} 30-\mathrm{C} 31-\mathrm{N} 4 & 0.5(2) \\ \mathrm{C} 5-\mathrm{C} 27-\mathrm{C} 28-\mathrm{C} 29 & 179.78(14) & \mathrm{C} 31-\mathrm{N} 4-\mathrm{C} 27-\mathrm{C} 5 & -179.19(13) \\ \mathrm{C} 6-\mathrm{N} 2-\mathrm{C} 10-\mathrm{C} 9 & 0.3(2) & \mathrm{C} 31-\mathrm{N} 4-\mathrm{C} 27-\mathrm{C} 28 & 0.7(2) \\ \mathrm{C} 6-\mathrm{C} 1-\mathrm{C} 2-\mathrm{C} 3 & 179.82(13) & & \end{array}$

Hydrogen-bond geometry $\left(\AA,{ }^{\circ}\right)$

$\mathrm{Cg}$ is the centroid of the $\mathrm{C} 11-\mathrm{C} 16$ ring.

\begin{tabular}{lllll}
\hline$D-\mathrm{H} \cdots A$ & $D-\mathrm{H}$ & $\mathrm{H} \cdots A$ & $D \cdots A$ & $D-\mathrm{H} \cdots A$ \\
\hline $\mathrm{C} 2-\mathrm{H} 2 \cdots \mathrm{N} 2$ & 0.95 & 2.50 & $2.815(2)$ & 99 \\
$\mathrm{C} 4-\mathrm{H} 4 \cdots \mathrm{N} 4$ & 0.95 & 2.46 & $2.778(2)$ & 100 \\
$\mathrm{C} 8-\mathrm{H} 8 \cdots{ }^{\mathrm{i}}$ & 0.95 & 2.59 & $3.529(2)$ & 170 \\
$\mathrm{C} 16-\mathrm{H} 16 \cdots \mathrm{Cg}^{\mathrm{ii}}$ & 0.95 & 2.81 & $3.669(2)$ & 151 \\
$\mathrm{C} 22-\mathrm{H} 22 \cdots{ }^{\mathrm{iii}}$ & 0.95 & 2.55 & $3.485(2)$ & 170
\end{tabular}

Symmetry codes: (i) $x-1, y, z+1$; (ii) $x,-y+1 / 2, z+1 / 2$; (iii) $x, y, z+1$. 